\title{
Contractions with rational inequalities in the extended $b$-metric space
}

\author{
Badr Alqahtani ${ }^{1}$, Andreea Fulga ${ }^{2}$, Erdal Karapınar ${ }^{3 *}$ (D) and Vladimir Rakočević ${ }^{4}$
}

\section{"Correspondence:}

erdalkarapinar@yahoo.com;

karapinar@mail.cmuh.org.tw

${ }^{3}$ Department of Medical Research,

China Medical University Hospital,

China Medical University, Taichung,

Taiwan

Full list of author information is

available at the end of the article

\begin{abstract}
In this paper, we prove some fixed point theorems for contractions of rational type in the setting of the extended $b$-metric spaces. We present some examples to illustrate the validity of our results. Our results improve and generalize a number of fixed point results in the literature.
\end{abstract}

MSC: 46T99; 47H10; 54H25

Keywords: Contractive mapping of type; Fixed point; Metric space

\section{Introduction and preliminaries}

Due to the wide application potential, one of the most discussed theorems in nonlinear analysis is the well-known Banach contraction principle. It has been generalized in several directions, such as, by relaxing the conditions of the abstract spaces, by relaxing the contraction types, and so on. Among them, we shall now mention the interesting papers of Dass and Gupta [3] and Jaggi [4] in which the rational type expressions were considered in the contraction condition (see also, e.g. [1-4, 6-9]). For the sake of completeness, we recollect the main results of these papers.

Theorem 1.1 ([4]) Let $(\mathcal{M}, d)$ be a complete metric space and $T: \mathcal{M} \rightarrow \mathcal{M}$ be a continuous mapping. If there exist $\alpha, \beta \in[0,1)$, with $\alpha+\beta<1$ such that

$$
d(T x, T y) \leq \alpha \cdot \frac{d(x, T x) d(y, T y)}{d(x, y)}+\beta \cdot d(x, y)
$$

for all distinct $x, y \in \mathcal{M}$, then $T$ possesses a unique fixed point in $\mathcal{M}$.

Theorem 1.2 ([3]) Let $(\mathcal{M}, d)$ be a complete metric space and $T: \mathcal{M} \rightarrow \mathcal{M}$ be a mapping. If there exist $\alpha, \beta \in[0,1)$, with $\alpha+\beta<1$ such that

$$
d(T x, T y) \leq \alpha \cdot d(y, T y) \frac{1+d(x, T x)}{1+d(x, y)}+\beta \cdot d(x, y)
$$

for all $x, y \in \mathcal{M}$, then $T$ has a unique fixed point $u \in X$. Moreover, the sequence $\left\{T^{n} x\right\}$ converges to the fixed point $u$ for all $x \in \mathcal{M}$.

(c) The Author(s) 2019. This article is distributed under the terms of the Creative Commons Attribution 4.0 International License (http://creativecommons.org/licenses/by/4.0/), which permits unrestricted use, distribution, and reproduction in any medium, provided you give appropriate credit to the original author(s) and the source, provide a link to the Creative Commons license, and indicate if changes were made. 
Throughout this paper, we shall denote the set of positive numbers and the set of real numbers by $\mathbb{N}$ and $\mathbb{R}$, respectively.

In this note, we shall reconsider the results of Dass and Gupta [3] and Jaggi [4] in a newly introduced abstract space, known as extended $b$-metric space. The notion of the extended $b$-metric space was introduced by Kamran et al. [5] as an extension of $b$-metric space.

Definition 1.1 ([5]) Let $\mathcal{M}$ be a nonempty set and $\theta: \mathcal{M} \times \mathcal{M} \rightarrow[1, \infty)$. A function $d_{\theta}: \mathcal{M} \times \mathcal{M} \rightarrow[0, \infty)$ is called an extended $b$-metric if for all $x, y, z \in \mathcal{M}$ it satisfies

$\left(d_{\theta} 1\right) \quad d_{\theta}(x, y)=0$ if and only if $x=y$;

$\left(d_{\theta} 2\right) \quad d_{\theta}(x, y)=d_{\theta}(y, x)$;

$\left(d_{\theta} 3\right) \quad d_{\theta}(x, z) \leq \theta(x, z)\left[d_{\theta}(x, y)+d_{\theta}(y, z)\right]$.

The pair $\left(\mathcal{M}, d_{\theta}\right)$ is called an extended $b$-metric space.

It is clear that an extended $b$-metric space coincides with the corresponding $b$-metric space, for $\theta(x, y)=s \geq 1$ where $s \in \mathbb{R}$ and it turns to be standard metric if $s=1$.

As expected, the basic topological notions were defined analogously.

Definition $1.2([5])$ Let $\left(\mathcal{M}, d_{\theta}\right)$ be an extended $b$-metric space.

(i) A sequence $x_{n}$ in $\mathcal{M}$ is said to converge to $x \in \mathcal{M}$, if for every $\epsilon>0$ there exists $N=N(\epsilon) \in \mathbb{N}$ such that $d_{\theta}\left(x_{n}, x\right)<\epsilon$, for all $n \geq N$. In this case, we write $\lim _{n \rightarrow \infty} x_{n}=x$.

(ii) A sequence $x_{n}$ in $\mathcal{M}$ is said to be Cauchy if for every $\epsilon>0$ there exists $N=N(\epsilon) \in \mathbb{N}$ such that $d_{\theta}\left(x_{m}, x_{n}\right)<\epsilon$, for all $m, n \geq N$.

Definition $1.3([5])$ An extended $b$-metric space $\left(\mathcal{M}, d_{\theta}\right)$ is complete if every Cauchy sequence in $\mathcal{M}$ is convergent.

Notice that an extended $b$-metric need not be continuous.

Lemma 1.1 ([5]) Let $\left(\mathcal{M}, d_{\theta}\right)$ be an extended b-metric space. If $d_{\theta}$ is continuous, then every convergent sequence has a unique limit.

For the sake of simplicity, throughout the paper, we assume that $\left(\mathcal{M}, d_{\theta}\right)$ represents a complete extended $b$-metric space. In addition, we assume that $d_{\theta}$ is a continuous functional unless otherwise stated.

In what follows, we recollect the main results of Kamran et al. [5] which is an analog of the Banach contraction principle in the context of extended $b$-metric space.

Theorem 1.3 ([5]) Let $T: \mathcal{M} \rightarrow \mathcal{M}$ be mapping. If there exists $\mathcal{K} \in[0,1)$ such that

$$
d_{\theta}(T x, T y) \leq \kappa \cdot d_{\theta}(x, y)
$$

for all $x, y \in \mathcal{M}$, where for each $x_{0} \in \mathcal{M}, \lim _{n, m \rightarrow \infty} \theta\left(x_{n}, x_{m}\right)<\frac{1}{k}$, where $x_{n}=T^{n} x_{0}, n \in \mathbb{N}$ Then $T$ has precisely one fixed point $u$. Moreover, for each $y \in \mathcal{M}, T^{n} y \rightarrow u$.

The main purpose of this paper is to extend the results of Kamran et al. [5] for the wellknown fixed point results, including the interesting theorems of Dass and Gupta [3] and Jaggi [4] in the frame of an extended $b$-metric space. 


\section{Main results}

Theorem 2.1 Let $T: \mathcal{M} \rightarrow \mathcal{M}$ be a continuous mapping such that, for all distinct $x, y \in$ $\mathcal{M}$,

$$
d_{\theta}(T x, T y) \leq \kappa \cdot R(x, y),
$$

where $K \in[0,1)$ and

$$
\begin{aligned}
\mathcal{R}(x, y)= & \max \left\{\frac{d_{\theta}(x, T x)\left[1+d_{\theta}(y, T y)\right]}{1+d_{\theta}(x, y)}, \frac{d_{\theta}(y, T y)\left[1+d_{\theta}(x, T x)\right]}{1+d_{\theta}(x, y)},\right. \\
& \left.\frac{d_{\theta}(y, T y) d_{\theta}(x, T x)}{d_{\theta}(x, y)}, d_{\theta}(x, y)\right\} .
\end{aligned}
$$

Suppose also that, for each $x_{0} \in \mathcal{M}, \lim _{n, m \rightarrow \infty} \theta\left(x_{n}, x_{m}\right)<\frac{1}{k}$, where $x_{n}=T^{n} x_{0}, n \in \mathbb{N}$. Then $T$ has a fixed point $u$. Moreover, for each $x \in \mathcal{M}$, we have $T^{n} x \rightarrow u$.

Proof By presumptions, for given $x_{0} \in \mathcal{M}$ we construct the sequence $\left\{x_{n}\right\}$ in $\mathcal{M}$ as $x_{n}=$ $T^{n} x_{0}=T x_{n-1}$, for $n \in \mathbb{N}$. If $x_{n_{0}}=x_{n_{0}+1}=T x_{n_{0}}$ for some $n_{0} \in \mathbb{N}_{0}:=\mathbb{N} \cup\{0\}$, then $x^{*}=x_{n_{0}}$ forms a fixed point for $T$ which completes the proof. Consequently, throughout the proof, we assume that

$$
x_{n} \neq x_{n+1} \quad \text { for all } n \in \mathbb{N}_{0} \text {. }
$$

By taking $x=x_{n-1}$ and $y=x_{n}$ in the inequality (4), we derive that

$$
d_{\theta}\left(x_{n}, x_{n+1}\right)=d_{\theta}\left(T x_{n-1}, T x_{n}\right) \leq \kappa \cdot R\left(x_{n-1}, x_{n}\right)
$$

with

$$
\begin{aligned}
\mathcal{R}\left(x_{n-1}, x_{n}\right) & =\max \left\{\begin{array}{c}
\frac{d_{\theta}\left(x_{n-1}, T x_{n-1}\right) d_{\theta}\left(x_{n}, T x_{n}\right)}{d_{\theta}\left(x_{n-1}, x_{n}\right)}, \frac{d_{\theta}\left(x_{n-1}, T x_{n-1}\right)\left[1+d_{\theta}\left(x_{n}, T x_{n}\right)\right]}{1+d_{\theta}\left(x_{n-1}, x_{n}\right)}, \\
\frac{d_{\theta}\left(x_{n}, T x_{n}\right)\left[1+d_{\theta}\left(x_{n-1}, T x_{n-1}\right)\right]}{1+d_{\theta}\left(x_{n-1}, x_{n}\right)}, d_{\theta}\left(x_{n-1}, x_{n}\right)
\end{array}\right\} \\
& =\max \left\{\begin{array}{c}
\frac{d_{\theta}\left(x_{n-1}, x_{n}\right) d_{\theta}\left(x_{n}, x_{n+1}\right)}{d_{\theta}\left(x_{n-1}, x_{n}\right)}, \frac{d_{\theta}\left(x_{n-1}, x_{n}\right)\left[1+d_{\theta}\left(x_{n}, x_{n+1}\right)\right]}{1+d_{\theta}\left(x_{n-1}, x_{n}\right)}, \\
\frac{d_{\theta}\left(x_{n}, x_{n+1}\right)\left[1+d_{\theta}\left(x_{n-1}, x_{n}\right)\right]}{1+d_{\theta}\left(x_{n-1}, x_{n}\right)}, d_{\theta}\left(x_{n-1}, x_{n}\right)
\end{array}\right\} \\
& =\max \left\{\frac{d_{\theta}\left(x_{n-1}, x_{n}\right)\left[1+d_{\theta}\left(x_{n}, x_{n+1}\right)\right]}{1+d_{\theta}\left(x_{n-1}, x_{n}\right)}, d_{\theta}\left(x_{n}, x_{n+1}\right), d_{\theta}\left(x_{n-1}, x_{n}\right)\right\} .
\end{aligned}
$$

Thus,

$$
d_{\theta}\left(x_{n}, x_{n+1}\right) \leq \kappa \max \left\{d_{\theta}\left(x_{n}, x_{n+1}\right), d_{\theta}\left(x_{n-1}, x_{n}\right), \frac{d_{\theta}\left(x_{n-1}, x_{n}\right)\left[1+d_{\theta}\left(x_{n}, x_{n+1}\right)\right]}{1+d_{\theta}\left(x_{n-1}, x_{n}\right)}\right\} .
$$

For refining the inequality above, we shall consider the following cases:

Case (i). If $\mathcal{R}\left(x_{n-1}, x_{n}\right)=d_{\theta}\left(x_{n}, x_{n+1}\right)$, then $d_{\theta}\left(x_{n}, x_{n+1}\right) \leq k \cdot d_{\theta}\left(x_{n}, x_{n+1}\right)$, which is a contradiction.

Case (ii). If $\mathcal{R}\left(x_{n-1}, x_{n}\right)=d_{\theta}\left(x_{n-1}, x_{n}\right)$, then the inequality (4) turns into the inequality below:

$$
0<d_{\theta}\left(x_{n}, x_{n+1}\right) \leq \kappa \cdot d_{\theta}\left(x_{n-1}, x_{n}\right) .
$$


Case (iii). Suppose that $\mathcal{R}\left(x_{n-1}, x_{n}\right)=\frac{d_{\theta}\left(x_{n-1}, x_{n}\right)\left[1+d_{\theta}\left(x_{n}, x_{n+1}\right)\right]}{1+d_{\theta}\left(x_{n-1}, x_{n}\right)}$; this yields

$$
\max \left\{d_{\theta}\left(x_{n-1}, x_{n}\right), d_{\theta}\left(x_{n}, x_{n+1}\right)\right\} \leq \frac{d_{\theta}\left(x_{n-1}, x_{n}\right)\left[1+d_{\theta}\left(x_{n}, x_{n+1}\right)\right]}{1+d_{\theta}\left(x_{n-1}, x_{n}\right)}
$$

We shall illustrate that this case is not possible. For this reason, we consider the following subcases:

Case (iii) $)_{a}$. Suppose that $\max \left\{d_{\theta}\left(x_{n-1}, x_{n}\right), d_{\theta}\left(x_{n}, x_{n+1}\right)\right\}=d_{\theta}\left(x_{n}, x_{n+1}\right)$, that is,

$$
d_{\theta}\left(x_{n-1}, x_{n}\right)<d_{\theta}\left(x_{n}, x_{n+1}\right)
$$

On the other hand, from (10), we have

$$
d_{\theta}\left(x_{n}, x_{n+1}\right) \leq \frac{d_{\theta}\left(x_{n-1}, x_{n}\right)\left[1+d_{\theta}\left(x_{n}, x_{n+1}\right)\right]}{1+d_{\theta}\left(x_{n-1}, x_{n}\right)}
$$

By a simple calculation, we derive, from the inequality above, that

$$
d_{\theta}\left(x_{n}, x_{n+1}\right) \leq d_{\theta}\left(x_{n-1}, x_{n}\right)
$$

which contradicts the assumption (11).

Case $(\mathrm{iii})_{b}$. Assume that $\max \left\{d_{\theta}\left(x_{n-1}, x_{n}\right), d_{\theta}\left(x_{n}, x_{n+1}\right)\right\}=d_{\theta}\left(x_{n-1}, x_{n}\right)$, that is,

$$
d_{\theta}\left(x_{n-1}, x_{n}\right)>d_{\theta}\left(x_{n}, x_{n+1}\right)
$$

Furthermore, from (10), we observe that

$$
d_{\theta}\left(x_{n-1}, x_{n}\right) \leq \frac{d_{\theta}\left(x_{n-1}, x_{n}\right)\left[1+d_{\theta}\left(x_{n}, x_{n+1}\right)\right]}{1+d_{\theta}\left(x_{n-1}, x_{n}\right)}
$$

A simple evaluation implies, from the inequality above, that

$$
d_{\theta}\left(x_{n-1}, x_{n}\right) \leq d_{\theta}\left(x_{n}, x_{n+1}\right)
$$

which contradicts the assumption (13). Hence, Case (iii) does not occur.

Consequently, we can state that the inequality (9) holds for all these three cases and by applying it recursively we obtain

$$
0<d_{\theta}\left(x_{n}, x_{n+1}\right) \leq \kappa^{n} d_{\theta}\left(x_{0}, x_{1}\right)
$$

Since $\mathcal{K} \in[0,1)$, we find that

$$
\lim _{n \rightarrow \infty} d_{\theta}\left(x_{n}, x_{n+1}\right)=0
$$

On the other hand, by $\left(d_{\theta} 3\right)$, together with the triangular inequality, for $p \geq 1$, we derive that

$$
\begin{aligned}
& d_{\theta}\left(x_{n}, x_{n+p}\right) \\
& \quad \leq \theta\left(x_{n}, x_{n+p}\right) \cdot\left[d_{\theta}\left(x_{n}, x_{n+1}\right)+d_{\theta}\left(x_{n+1}, x_{n+p}\right)\right]
\end{aligned}
$$




$$
\begin{aligned}
\leq & \theta\left(x_{n}, x_{n+p}\right) d_{\theta}\left(x_{n}, x_{n+1}\right)+\theta\left(x_{n}, x_{n+p}\right) d_{\theta}\left(x_{n+1}, x_{n+p}\right) \\
\leq & \theta\left(x_{n}, x_{n+p}\right) \kappa^{n} d_{\theta}\left(x_{0}, x_{1}\right)+\theta\left(x_{n}, x_{n+p}\right) \theta\left(x_{n+1}, x_{n+p}\right)\left[d_{\theta}\left(x_{n+1}, x_{n+2}\right)+d_{\theta}\left(x_{n+2}, x_{n+p}\right)\right] \\
\leq & \theta\left(x_{n}, x_{n+p}\right) \cdot \kappa^{n} d_{\theta}\left(x_{0}, x_{1}\right)+\theta\left(x_{n}, x_{n+p}\right) \theta\left(x_{n+1}, x_{n+p}\right) \cdot \kappa^{n+1} d_{\theta}\left(x_{0}, x_{1}\right)+\cdots \\
& +\theta\left(x_{n}, x_{n+p}\right) \cdots \theta \theta\left(x_{n+p-1}, x_{n+p}\right) \cdot k^{n+p-1} d_{\theta}\left(x_{0}, x_{1}\right) \\
= & d_{\theta}\left(x_{0}, x_{1}\right) \sum_{i=1}^{n+p-1} \kappa^{i} \prod_{j=1}^{i} \theta\left(x_{n+j}, x_{n+p}\right) .
\end{aligned}
$$

Notice the inequality above is dominated by $\sum_{i=1}^{n+p-1} \kappa^{i} \prod_{j=1}^{i} \theta\left(x_{n+j}, x_{n+p}\right) \leq \sum_{i=1}^{n+p-1} \kappa^{i} \times$ $\prod_{j=1}^{i} \theta\left(x_{j}, x_{n+p}\right)$.

On the other hand, by employing the ratio test, we conclude that the series $\sum_{i=1}^{\infty} k^{i} \times$ $\prod_{j=1}^{i} \theta\left(x_{j}, x_{n+p}\right)$ converges to some $S \in(0, \infty)$. Indeed, $\lim _{i \rightarrow \infty} \frac{a_{i+1}}{a_{i}}=\lim _{i \rightarrow \infty} k \theta\left(x_{i}, x_{i+p}\right)<1$ and hence we get the desired result. Thus, we have

$$
S=\sum_{i=1}^{\infty} \kappa^{i} \prod_{j=1}^{i} \theta\left(x_{j}, x_{n+p}\right) \quad \text { with the partial sum } S_{n}=\sum_{i=1}^{n} \kappa^{i} \prod_{j=1}^{i} \theta\left(x_{j}, x_{n+p}\right) .
$$

Consequently, we observe, for $n \leq 1, p \leq 1$, that

$$
d_{\theta}\left(x_{n}, x_{n+p}\right) \leq k^{n} d_{\theta}\left(x_{0}, x_{1}\right)\left[S_{n+p-1}-S_{n-1}\right] .
$$

Letting $n \rightarrow \infty$ in (18), we conclude that the constructed sequence $\left\{x_{n}\right\}$ is Cauchy in the extended $b$-metric space $\left(\mathcal{M}, d_{\theta}\right)$. Regarding the assumption of the completeness, we conclude that there exists $u \in \mathcal{M}$ such that $x_{n} \rightarrow u$ as $n \rightarrow \infty$. Due to the continuity of $T$, we shall show that the limit point $u$ is a fixed point of $T$. Indeed, we have

$$
T u=T\left(\lim _{n \rightarrow \infty} x_{n}\right)=\lim _{n \rightarrow \infty} T\left(x_{n}\right)=\lim _{n \rightarrow \infty} x_{n+1}=u .
$$

Corollary 2.1 A continuous mapping $T: \mathcal{M} \rightarrow \mathcal{M}$ has a fixed point provided that, for all distinct $x, y \in \mathcal{M}$,

$$
\begin{aligned}
d_{\theta}(T x, T y) \leq & a_{1} \frac{d_{\theta}(x, T x)\left[1+d_{\theta}(y, T y)\right]}{1+d_{\theta}(x, y)}+a_{2} \frac{d_{\theta}(y, T y)\left[1+d_{\theta}(x, T x)\right]}{1+d_{\theta}(x, y)} \\
& +a_{3} \frac{d_{\theta}(x, T x) d_{\theta}(y, T y)}{d_{\theta}(x, y)}+a_{4} d_{\theta}(x, y),
\end{aligned}
$$

where $a_{i} \in[0,1), i \in\{1,2,3,4\}$ with $\sum_{i=1}^{4} a_{i}<1$ and for each $x_{0} \in \mathcal{M}, \lim _{n, m \rightarrow \infty} \theta\left(x_{n}, x_{m}\right)<$ $\frac{1}{\sum_{i=1}^{4} a_{i}}$, where $x_{n}=T^{n} x_{0}, n \in \mathbb{N}$.

Proof The proof follows from Theorem 2.1 by letting $k=\sum_{i=1}^{4} a_{i}$. Indeed, we have

$$
\begin{aligned}
d_{\theta}(T x, T y) \leq & a_{1} \frac{d_{\theta}(x, T x)\left[1+d_{\theta}(y, T y)\right]}{1+d_{\theta}(x, y)} \\
& +a_{2} \frac{d_{\theta}(y, T y)\left[1+d_{\theta}(x, T x)\right]}{1+d_{\theta}(x, y)}
\end{aligned}
$$




$$
\begin{aligned}
& +a_{3} \frac{d_{\theta}(x, T x) d_{\theta}(y, T y)}{d_{\theta}(x, y)}+a_{4} d_{\theta}(x, y) \\
\leq & \left(a_{1}+a_{2}+a_{3}+a_{4}\right) \cdot R(x, y) .
\end{aligned}
$$

Regarding the analogy, we skip the details.

Corollary 2.2 Let $T: \mathcal{M} \rightarrow \mathcal{M}$ be a continuous mapping. Suppose that, for all distinct $x, y \in \mathcal{M}$, we have the inequality

$$
d_{\theta}(T x, T y) \leq \alpha \cdot \frac{d_{\theta}(x, T x) d_{\theta}(y, T y)}{d_{\theta}(x, y)}+\beta d_{\theta}(x, y)
$$

where $\alpha, \beta \in[0,1), \alpha+\beta<1$. Suppose also that, for each $x_{0} \in \mathcal{M}, \lim _{n, m \rightarrow \infty} \theta\left(x_{n}, x_{m}\right)<\frac{1}{\alpha+\beta}$, where $x_{n}=T^{n} x_{0}, n \in \mathbb{N}$. Then $T$ has a unique fixed point $u$. Moreover, for each $x \in \mathcal{M}$, we have $T^{n} x \rightarrow u$.

Proof The proof follows from Corollary 2.1 by letting $k=\alpha+\beta$, where $a_{1}=a_{2}=0$ and $a_{3}=\alpha, a_{4}=\beta$.

Example 2.1 Let $\left(\mathcal{M}, d_{\theta}\right)$ be a complete extended $b$-metric space, where $\mathcal{M}=[0, \infty)$ and $d_{\theta}: \mathcal{M} \times \mathcal{M} \rightarrow[0, \infty), d_{\theta}(x, y)=(x-y)^{2}$ and $\theta: \mathcal{M} \times \mathcal{M} \rightarrow[1, \infty)$ is defined as $\theta(x, y)=$ $x+y+2$. Let $T: \mathcal{M} \rightarrow \mathcal{M}$ be defined by $T x=\frac{x}{3}$. Obviously,

$$
d_{\theta}(T x, T y)=\frac{(x-y)^{2}}{9}, \quad d_{\theta}(x, T x)=\frac{4 x^{2}}{9}, \quad d_{\theta}(y, T y)=\frac{4 y^{2}}{9}
$$

and, choosing $\alpha=\frac{2}{9}$ and $\beta=\frac{1}{9}$, we have

$$
\begin{aligned}
\lim _{n \rightarrow \infty} \theta\left(x_{n}, x_{n+p}\right) & =\lim _{n \rightarrow \infty} \theta\left(T^{n} x, T^{n+p} x\right) \\
& =\lim _{n \rightarrow \infty} \theta\left(\frac{x}{3^{n}}, \frac{x}{3^{n+p}}\right) \\
& =\lim _{n \rightarrow \infty}\left(\frac{x}{3^{n}}+\frac{x}{3^{n+p}}+2\right)<3=\frac{1}{\alpha+\beta} .
\end{aligned}
$$

By routine calculation, we obtain

$$
\begin{aligned}
d_{\theta}(T x, T y) & =\frac{(x-y)^{2}}{9} \leq \frac{1}{3}(x-y)^{2} \leq \frac{2}{9} \cdot \frac{\frac{4 x^{2}}{9} \cdot \frac{4 y^{2}}{9}}{(x-y)^{2}}+\frac{1}{9}(x-y)^{2} \\
& =\frac{2}{9} \cdot \frac{d_{\theta}(x, T x) d_{\theta}(y, T y)}{d_{\theta}(x, y)}+\frac{1}{9} d_{\theta}(x, y) .
\end{aligned}
$$

Therefore, all conditions of Corollary 2.2 are satisfied. Thus, $T$ has a fixed point.

Corollary 2.3 Let $T: \mathcal{M} \rightarrow \mathcal{M}$ be a mapping such that, for all $x, y \in \mathcal{M}$ we have

$$
d_{\theta}(T x, T y) \leq \alpha \cdot \frac{d_{\theta}(y, T y)\left[1+d_{\theta}(x, T x)\right]}{1+d_{\theta}(x, y)}+\beta \cdot d_{\theta}(x, y),
$$


where $\alpha, \beta \in[0,1), \alpha+\beta<1$. Suppose also that, for each $x_{0} \in \mathcal{M}, \lim _{n, m \rightarrow \infty} \theta\left(x_{n}, x_{m}\right)<\frac{1}{\alpha+\beta}$, where $x_{n}=T^{n} x_{0}, n \in \mathbb{N}$. Then $T$ has a unique fixed point $u$. Moreover, for each $x \in \mathcal{M}$, we have $T^{n} x \rightarrow u$.

Proof Letting $\mathcal{K}=\alpha+\beta$, where $a_{1}=a_{3}=0$ and $a_{2}=\alpha, a_{4}=\beta$ in Corollary 2.1, and following the steps of the proof of Theorem 2.1, we know that there exists $u \in \mathcal{M}$ such that $T^{n} x \rightarrow u$. We must prove that this point is the unique fixed point of $T$. Indeed,

$$
\begin{aligned}
d_{\theta}(T u, u) & \leq \theta(T u, u) \cdot\left[d_{\theta}\left(T u, T x_{n}\right)+d_{\theta}\left(T x_{n}, u\right)\right] \\
& \leq \theta(T u, u)\left[\alpha \cdot \frac{d_{\theta}\left(x_{n}, x_{n+1}\right)\left(1+d_{\theta}(u, T u)\right.}{1+d_{\theta}\left(u, x_{n}\right)}+\beta \cdot d_{\theta}\left(u, x_{n}\right)+d_{\theta}\left(x_{n+1}, u\right)\right] .
\end{aligned}
$$

Letting $n \rightarrow \infty$ in the above inequality we get $d_{\theta}(T u, u)=0$. Hence $T u=u$.

In order to show the uniqueness, suppose that there exists $v \in \mathcal{M}$ such that $T u=u \neq v=$ Tv. Then

$$
\begin{aligned}
d_{\theta}(u, v) & =d_{\theta}(T u, T v) \\
& \leq \alpha \cdot \frac{d_{\theta}(v, T v)\left(1+d_{\theta}(u, T u)\right.}{1+d_{\theta}(u, v)}+\beta \cdot d_{\theta}(u, v) \\
& =\beta \cdot d_{\theta}(u, v)<d_{\theta}(u, v),
\end{aligned}
$$

which is a contradiction. Thus, we have completed the proof.

Example 2.2 Let $\mathcal{M}=\{1,2,3,4, \ldots\}$ and define $d_{\theta}: \mathcal{M} \times \mathcal{M} \rightarrow[0, \infty)$ as

$$
d_{\theta}(x, y)=(x-y)^{4},
$$

where $\theta: \mathcal{M} \times \mathcal{M} \rightarrow[1, \infty)$ is a function defined by

$$
\theta(x, y)= \begin{cases}|x-y|^{3}, & \text { if } x \neq y \\ 1, & \text { if } x=y\end{cases}
$$

Then $\left(\mathcal{M}, d_{\theta}\right)$ forms an extended b-metric space (see Example 3.1 in [10]).

Let $T: \mathcal{M} \rightarrow \mathcal{M}$ be defined by

$$
T x= \begin{cases}3, & \text { if } x=1 \\ 4, & \text { otherwise. }\end{cases}
$$

Let also $\alpha=\frac{1}{16}$ and $\beta=\frac{1}{8}$. Of course, since $d_{\theta}(x, 1)=(x-1)^{4} \geq 2^{4}$ and $d_{\theta}(T x, T 1)=1$ for any $x \in\{3,4,5, \ldots\}$, the inequality (22) becomes

$$
d_{\theta}(T x, T 1)=1<\frac{1}{8} 2^{4} \leq \frac{1}{8}(x-1)^{4} \leq \alpha \cdot \frac{d_{\theta}(1, T 1)\left[1+d_{\theta}(x, T x)\right]}{1+d_{\theta}(x, 1)}+\beta \cdot d_{\theta}(x, 1) .
$$


For the case $x=2$ and $y=1$, we have $d_{\theta}(2,1)=1$ and $\frac{d_{\theta}(1, T 1)\left[1+d_{\theta}(2, T 2)\right]}{1+d_{\theta}(2,1)}=\frac{2^{4}\left(1+2^{4}\right)}{2}$. Obviously, we have

$$
d_{\theta}(T 2, T 1)=1 \leq \frac{1}{16} \cdot \frac{2^{4}\left(1+2^{4}\right)}{2}+\frac{1}{8}=\alpha \cdot \frac{d_{\theta}(1, T 1)\left[1+d_{\theta}(2, T 2)\right]}{1+d_{\theta}(2,1)}+\beta \cdot d_{\theta}(2,1)
$$

For all other cases, $d_{\theta}(T x, T y)=0$ and the existence of a fixed point is ensured by Corollary 2.3.

Corollary 2.4 Theorem 1.3 is concluded from Corollary 2.2 and hence Theorem 2.1.

Proof It is sufficient to take $\alpha=0$.

\subsection{Removing the necessity of the continuity of the functional $d_{\theta}$}

In the following theorem, we relax the condition by removing the continuity of the functional $d_{\theta}$ in the following setting.

Theorem 2.2 Let $T: \mathcal{M} \rightarrow \mathcal{M}$ be a mapping that satisfies the inequality

$$
d_{\theta}(T x, T y) \leq \kappa \cdot \max \left\{\frac{d_{\theta}(x, T x) d_{\theta}(x, T y)+d_{\theta}(y, T y) d_{\theta}(y, T x)}{d_{\theta}(x, T y)+d_{\theta}(y, T x)}, d_{\theta}(x, y)\right\}
$$

for all $x, y \in \mathcal{M}$, where $\kappa \in[0,1)$, be such that, for each $x_{0} \in \mathcal{M}, \lim _{n, m \rightarrow \infty} \theta\left(x_{n}, x_{m}\right)<\frac{1}{\kappa}$, where $x_{n}=T^{n} x_{0}, n \in \mathbb{N}$. Then $T$ has a unique fixed point $u$. Moreover, for each $y \in X$, $T^{n} y \rightarrow u$.

Proof By taking $x=x_{n-1}$ and $y=x_{n}$ in the inequality (24), we get

$$
\begin{aligned}
0 & <d_{\theta}\left(x_{n}, x_{n+1}\right)=d_{\theta}\left(T x_{n-1}, T x_{n}\right) \\
& \leq \mathcal{K} \cdot \max \left\{\frac{d_{\theta}\left(x_{n-1}, x_{n}\right) d_{\theta}\left(x_{n-1}, x_{n+1}\right)+d_{\theta}\left(x_{n}, x_{n+1}\right) d_{\theta}\left(x_{n}, x_{n}\right)}{d_{\theta}\left(x_{n-1}, x_{n+1}\right)+d_{\theta}\left(x_{n}, x_{n}\right)}, d_{\theta}\left(x_{n-1}, x_{n}\right)\right\} \\
& =\mathcal{K} \cdot \max \left\{\frac{d_{\theta}\left(x_{n-1}, x_{n}\right) d_{\theta}\left(x_{n-1}, x_{n+1}\right)}{d_{\theta}\left(x_{n-1}, x_{n+1}\right)}, d_{\theta}\left(x_{n-1}, x_{n}\right)\right\} \\
& =\mathcal{K} \cdot d_{\theta}\left(x_{n-1}, x_{n}\right) .
\end{aligned}
$$

Recursively, we derive that

$$
d_{\theta}\left(x_{n}, x_{n+1}\right) \leq k^{n} d_{\theta}\left(x_{0}, x_{1}\right), \quad \forall n \in \mathbb{N}
$$

and regarding that $0 \leq k<1$, we derive that

$$
\lim _{n \rightarrow \infty} d_{\theta}\left(x_{n}, x_{n+1}\right)=0
$$

On the other hand, by following the same lines in the previous theorem, we conclude that $\left\{x_{n}\right\}$ is a Cauchy sequence. Since $\mathcal{M}$ is complete, there exists $u \in \mathcal{M}$ such that the sequence $\left\{x_{n}=T^{n} x_{0}\right\}$ converges to $u$, that is,

$$
\lim _{n \rightarrow \infty} d_{\theta}\left(x_{n}, u\right)=0
$$


As a next step, we shall prove that $u$ is a fixed point of $T$. By using (24) and the triangle inequality, we have

$$
\begin{aligned}
d_{\theta}(u, T u) \leq & \theta(u, T u) \cdot\left[d_{\theta}\left(u, T x_{n}\right)+d_{\theta}\left(T x_{n}, T u\right)\right] \\
\leq & \theta(u, T u) \cdot\left[d_{\theta}\left(u, x_{n+1}\right)\right. \\
& \left.+\kappa \cdot \max \left\{\frac{d_{\theta}(u, T u) d_{\theta}\left(u, x_{n+1}\right)+d_{\theta}\left(x_{n}, x_{n+1}\right) d_{\theta}\left(x_{n}, T u\right)}{d_{\theta}\left(u, x_{n+1}\right)+d_{\theta}\left(x_{n}, T u\right)}, d_{\theta}\left(u, x_{n}\right)\right\}\right] .
\end{aligned}
$$

Letting $n \rightarrow \infty$, and taking (27) and (28) into account, we get

$$
d_{\theta}(T u, u) \leq 0
$$

Accordingly, we have $d_{\theta}(T u, u)=0$, that is, $T u=u$.

Lastly, we shall indicate that this fixed point is unique. Suppose, on the contrary, that it is not unique. Thus, there exists another fixed point $v$ of $T$ that is distinct from $u$. Now, by using (24) and $k<1$,

$$
\begin{aligned}
0 & <d_{\theta}(u, v)=d_{\theta}(T u, T v) \\
& \leq \mathcal{K} \cdot \max \left\{\frac{d_{\theta}(u, u) d_{\theta}(u, v)+d_{\theta}(v, v) d_{\theta}(v, u)}{d_{\theta}(u, v)+d_{\theta}(u, v)}, d_{\theta}(u, v)\right\} \\
& =\mathcal{K} \cdot d_{\theta}(u, v)<d_{\theta}(u, v),
\end{aligned}
$$

which shows that $u=v$. Therefore, $T$ has a unique fixed point.

Example 2.3 Let $\mathcal{M}=\left\{\frac{1}{2}, \frac{1}{4}, \frac{1}{8}\right\}$ and the functions $\theta: \mathcal{M} \times \mathcal{M} \rightarrow[1, \infty), d_{\theta}: \mathcal{M} \times \mathcal{M} \rightarrow$ $[0, \infty)$ be defined as $\theta(x, y)=x+y+1$ and

$$
\begin{aligned}
& d_{\theta}\left(\frac{1}{2}, \frac{1}{4}\right)=d_{\theta}\left(\frac{1}{4}, \frac{1}{2}\right)=1, \quad d_{\theta}\left(\frac{1}{2}, \frac{1}{8}\right)=d_{\theta}\left(\frac{1}{8}, \frac{1}{2}\right)=2, \\
& d_{\theta}\left(\frac{1}{8}, \frac{1}{4}\right)=d_{\theta}\left(\frac{1}{4}, \frac{1}{8}\right)=3, \\
& d_{\theta}\left(\frac{1}{2}, \frac{1}{2}\right)=d_{\theta}\left(\frac{1}{4}, \frac{1}{4}\right)=d_{\theta}\left(\frac{1}{8}, \frac{1}{8}\right)=0 .
\end{aligned}
$$

Let $\mathcal{K}=\frac{7}{12}$ and $T: \mathcal{M} \rightarrow \mathcal{M}$ be defined by

$$
T \frac{1}{2}=T \frac{1}{4}=\frac{1}{4} \quad \text { and } \quad T \frac{1}{8}=\frac{1}{2}
$$

We have $T^{n} x \rightarrow 0$ for any $x \in \mathcal{M}$ and $\lim _{n, m \rightarrow \infty} \theta\left(T^{n} x, T^{m} x\right)=\frac{1}{4}+\frac{1}{4}+1<\frac{3}{2}<\frac{12}{7}=\frac{1}{k}$.

But

$$
\begin{aligned}
& \theta\left(\frac{1}{2}, \frac{1}{4}\right)=\frac{1}{2}+\frac{1}{4}+1=\frac{7}{4}, \\
& \theta\left(\frac{1}{2}, \frac{1}{8}\right)=\frac{1}{2}+\frac{1}{8}+1=\frac{13}{8},
\end{aligned}
$$




$$
\theta\left(\frac{1}{8}, \frac{1}{4}\right)=\frac{1}{8}+\frac{1}{4}+1=\frac{11}{8}
$$

and

$$
\begin{aligned}
& 1=d_{\theta}\left(\frac{1}{2}, \frac{1}{4}\right) \leq \theta\left(\frac{1}{2}, \frac{1}{4}\right)\left(d_{\theta}\left(\frac{1}{2}, \frac{1}{8}\right)+d_{\theta}\left(\frac{1}{8}, \frac{1}{4}\right)\right)=\frac{7}{4}(2+3), \\
& 2=d_{\theta}\left(\frac{1}{2}, \frac{1}{8}\right) \leq \theta\left(\frac{1}{2}, \frac{1}{8}\right)\left(d_{\theta}\left(\frac{1}{2}, \frac{1}{4}\right)+d_{\theta}\left(\frac{1}{8}, \frac{1}{4}\right)\right)=\frac{13}{8}(1+3)=\frac{13}{2}, \\
& 3=d_{\theta}\left(\frac{1}{4}, \frac{1}{8}\right) \leq \theta\left(\frac{1}{4}, \frac{1}{8}\right)\left(d_{\theta}\left(\frac{1}{4}, \frac{1}{2}\right)+d_{\theta}\left(\frac{1}{2}, \frac{1}{8}\right)\right)=\frac{11}{8}(1+2)=\frac{33}{8},
\end{aligned}
$$

which proves that $d_{\theta}$ is an extended $b$-metric on $\mathcal{M}$.

We will consider the following cases:

(i) For $x=\frac{1}{2}, y=\frac{1}{4}$, we have $d_{\theta}\left(T \frac{1}{2}, T \frac{1}{4}\right)=d_{\theta}\left(\frac{1}{4}, \frac{1}{4}\right)=0$, so the condition from

Theorem 2.2 is satisfied.

(ii) For $x=\frac{1}{2}, y=\frac{1}{8}$

$$
\begin{aligned}
1 & =d_{\theta}\left(T \frac{1}{2}, T \frac{1}{8}\right) \\
& \leq \kappa \max \left\{\frac{d_{\theta}\left(\frac{1}{2}, T \frac{1}{2}\right) d_{\theta}\left(\frac{1}{2}, T \frac{1}{8}\right)+d_{\theta}\left(\frac{1}{8}, T \frac{1}{8}\right) d_{\theta}\left(\frac{1}{8}, T \frac{1}{2}\right)}{d_{\theta}\left(\frac{1}{2}, T \frac{1}{8}\right)+d_{\theta}\left(\frac{1}{8}, T \frac{1}{2}\right)}, d_{\theta}\left(\frac{1}{2}, \frac{1}{8}\right)\right\} \\
& =\frac{7}{12} \max \left\{\frac{0+2 \cdot 3}{1+2}, 2\right\}=\frac{7}{6} .
\end{aligned}
$$

(iii) For $x=\frac{1}{4}, y=\frac{1}{8}$

$$
\begin{aligned}
1 & =d_{\theta}\left(T \frac{1}{4}, T \frac{1}{8}\right) \\
& \leq \kappa \max \left\{\frac{d_{\theta}\left(\frac{1}{4}, T \frac{1}{4}\right) d_{\theta}\left(\frac{1}{4}, T \frac{1}{8}\right)+d_{\theta}\left(\frac{1}{8}, T \frac{1}{8}\right) d_{\theta}\left(\frac{1}{8}, T \frac{1}{4}\right)}{d_{\theta}\left(\frac{1}{4}, T \frac{1}{8}\right)+d_{\theta}\left(\frac{1}{8}, T \frac{1}{4}\right)}, d_{\theta}\left(\frac{1}{4}, \frac{1}{8}\right)\right\} \\
& =\frac{7}{12} \max \left\{\frac{0+2 \cdot 3}{1+3}, 3\right\}=\frac{7}{4} .
\end{aligned}
$$

Therefore all conditions of Theorem 2.2 are satisfied. Hence $T$ has a unique fixed point, $x=\frac{1}{4}$.

Corollary 2.5 Let $T: \mathcal{M} \rightarrow \mathcal{M}$ be a mapping that satisfies

$$
d_{\theta}(T x, T y) \leq \alpha \cdot \frac{d_{\theta}(x, T x) d_{\theta}(x, T y)+d_{\theta}(y, T y) d_{\theta}(y, T x)}{d_{\theta}(x, T y)+d_{\theta}(y, T x)}+\beta \cdot d_{\theta}(x, y)
$$

for all $x, y \in \mathcal{M}$, where $\alpha, \beta \in[0,1), \alpha+\beta<1$ are such that, for each $x_{0} \in \mathcal{M}$, $\lim _{n, m \rightarrow \infty} \theta\left(x_{n}, x_{m}\right)<\frac{1}{\alpha+\beta}$, where $x_{n}=T^{n} x_{0}, n \in \mathbb{N}$. Then $T$ has a unique fixed point $u$. Moreover, for each $x \in \mathcal{M}, T^{n} x \rightarrow u$.

\section{Conclusion}

It is clear that the corresponding results in the setting of both $b$-metric space and standard metric space can be included in our results, by letting $\theta(x, y)=s \geq 1$ and $\theta(x, y)=1$, 
respectively, in the related places. In particular, the main results of Dass and Gupta [3], Jaggi [4] and also the well-known Banach contraction mapping principle are derived from our results.

Funding

Not applicable.

Competing interests

The authors declare that they have no competing interests.

Authors' contributions

All authors contributed equally and significantly in writing this article. All authors read and approved the final manuscript.

\section{Author details}

${ }^{1}$ Department of Mathematics, King Saud University, Riyadh, Saudi Arabia. ${ }^{2}$ Department of Mathematics and Computer Sciences, Universitatea Transilvania Brasov, Brasov, Romania. ${ }^{3}$ Department of Medical Research, China Medical University Hospital, China Medical University, Taichung, Taiwan. ${ }^{4}$ Faculty of Sciences and Mathematics, University of Niš, Niš, Serbia.

\section{Publisher's Note}

Springer Nature remains neutral with regard to jurisdictional claims in published maps and institutional affiliations.

Received: 10 June 2019 Accepted: 7 August 2019 Published online: 20 August 2019

References

1. Arshad, M., Karapınar, E., Jamshaid, A.: Some unique fixed point theorems for rational contractions in partially ordered metric spaces. J. Inequal. Appl. 2013, Article ID 248 (2013)

2. Chandok, S., Karapinar, E.: Common fixed point of generalized rational type contraction mappings in partially ordered metric spaces. Thai J. Math. 11(2), 251-260 (2013)

3. Dass, B.K., Gupta, S.: An extension of Banach contraction principle through rational expressions. Indian J. Pure Appl. Math. 6, 1455-1458 (1975)

4. Jaggi, D.S.: Some unique fixed point theorems. Indian J. Pure Appl. Math. 8, 223-230 (1977)

5. Kamran, T., Samreen, M., Ain, O.U.: A generalization of $b$-metric space and some fixed point theorems. Mathematics $\mathbf{5}$, 19 (2017)

6. Karapinar, E., Marudai, M., Pragadeeswarar, V.: Fixed point theorems for generalized weak contractions satisfying rational expression on a ordered partial metric space. Lobachevskii J. Math. 34(1), 116-123 (2013)

7. Karapinar, E., Roldan, A., Sadarangani, K.: Existence and uniqueness of best proximity points under rational contractivity conditions. Math. Slovaca 66(6), 1427-1442 (2016)

8. Karapinar, E., Shatanawi, W., Tas, K.: Fixed point theorem on partial metric spaces involving rational expressions. Miskolc Math. Notes 14(1), 135-142 (2013)

9. Mustafa, Z., Karapınar, E., Aydi, H.: A discussion on generalized almost contractions via rational expressions in partially ordered metric spaces. J. Inequal. Appl. 2014, 219 (2014)

10. Samreen, M., Kamran, T., Postolache, M.: Extended $b$-metric space, extended $b$-comparison function and nonlinear contractions. Sci. Bull. "Politeh." Univ. Buchar., Ser. A, Appl. Math. Phys. 80(4), $21-28$ (2018)

\section{Submit your manuscript to a SpringerOpen ${ }^{\circ}$ journal and benefit from:}

- Convenient online submission

- Rigorous peer review

- Open access: articles freely available online

- High visibility within the field

Retaining the copyright to your article 